Voix et Images

voixetimages

\title{
Le corps à corps passionné
}

\section{Robert Major}

Volume 17, numéro 3 (51), printemps 1992

Paul-Marie Lapointe

URI : https://id.erudit.org/iderudit/200983ar

DOI : https://doi.org/10.7202/200983ar

Aller au sommaire du numéro

\section{Éditeur(s)}

Université du Québec à Montréal

\section{ISSN}

0318-9201 (imprimé)

1705-933X (numérique)

Découvrir la revue

\section{Citer cet article}

Major, R. (1992). Le corps à corps passionné. Voix et Images, 17(3), 512-520.

https://doi.org/10.7202/200983ar d'utilisation que vous pouvez consulter en ligne.

https://apropos.erudit.org/fr/usagers/politique-dutilisation/ 


\title{
Essai
}

\section{Le corps à corps passionné}

\author{
Robert Major, Université d'Ottawa
}

La critique pour autant qu'elle est un corps à corps avec le texte.

André Brochu

Je n'ai jamais abordé une problématique littéraire ou parlé d'un texte de poésie sans y mettre de la passion.

Joseph Bonenfant

Je n'aime guère les épigraphes. Joseph Bonenfant me pardonnera, lui qui choisit de donner des chapeaux épigraphiques aux parties de son livre et qui se défend bien d'en faire une utilisation autoritaire ou complaisante. Rien n'y fait. Ces sentinelles à la marge du texte, qui m'interpellent avec solennité avant que je ne franchisse son portail, m'intimident et, je l'avoue, m'agacent quelque peu. Marqueurs de tonalité, certes, mais avec un soupçon d'autocratie qui laisse songeur. D'entrée de jeu les épigraphes nous flanquent à la figure une phrase, sinon deux ou trois, habituellement d'auteurs différents, tous à la mode, bien entendu, et reconnus pour leur hermétisme ou leur "profondeur ". Et elles nous disent, un peu trop vivement: voici, en incipit, un texte privilégié, lourd de sens, prégnant de significations graves, abîme à sonder avant d'aborder le texte propre, qui en est le décalage ou la simple excroissance. Jeu spéculaire, de mise il n'y a pas si longtemps, encore endémique dans certains milieux, et dont l'effet est plutôt ambigu, quelquefois agaçant. À la limite, on pourrait se contenter de ces phrases en médaillon et se passer du texte en appendice!

Mais l'exemple est contagieux et j'y succombe. Une fois n'est pas coutume. D'ailleurs, qui pourrait nier l'indicible plaisir qu'on trouve à se contredire soi-même! Je tire donc mes épigraphes des derniers livres d'André Brochu et de Joseph Bonenfant, recueils d'études avec de très beaux titres et des contenus à l'avenant: Le Singulier pluriel ${ }^{1}$ et Passions du poétique ${ }^{2}$. Ces tronçons de phrase me semblent condenser 
avec bonheur une vision de la critique et du travail littéraire aux antipodes de celle véhiculée par les instances subventionnaires et cautionnée par l'institution universitaire, cette dernière elle aussi, hélas! carrément obnubilée par le modèle scientifique. Ces épigraphes, et les livres dont elles sont issues, proposent une vision du travail critique difficilement comptabilisable; vision qui n'implique que le littéraire (sans équipe!), seul, tout nu devant l'œuvre, la feuille blanche sous la main; vision réfractaire, on le voit, aux catégories de la "recherche" dont les canons et les critères nous sont de plus en plus imposés par les sciences * pures. (et dures).

Vision rétrograde? Essentielle, plutôt, et fondement à ce qui s'accomplit, par ailleurs, avec budget, équipes et train. Fondement et but ultime. Il faut bien, en effet, que quelqu'un lise les œuvres. Une fois qu'on les a répertoriées, classées, fichées et intégrées dans force dictionnaires, bibliographies ou histoires littéraires, (tous indispensables, certes, là n'est pas la question!), avant et pendant qu'on les ressasse ainsi, et après qu'on les a cataloguées, il faut bien qu'un lecteur les recrée, les fasse parler, les fasse reparler. Que dans un acte d'écriture, qui est aussi générateur de savoir, et de la plus haute tenue, un critique se propose comme médiateur entre l'œuvre et d'autres lecteurs.

Comme complément (contrepoids?), donc, à ma dernière chronique sur les effets remarquables des équipes de recherche, voici quelques littéraires, d'une autre constitution, et dont on ne saurait, non plus, se passer.

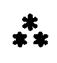

Sur ce genre particulier d'essai qu'est le rapaillage de textes divers, sur l'intérêt et l'utilité de ces livres bricolés - à peu de frais, pourraiton croire - à partir de pièces éparses, sur ces exemples de recyclagé intellectuel, je ne crois pas devoir revenir, m'étant déjà compromis en rendant compte de quelques titres de la collection *Papiers collés a au Boréal (Voix E Images, $\mathrm{n}^{\circ} 42$ ) - collection vouée à cette opération et où André Brochu a aussi publié, d'ailleurs - et n'ayant pas changé d'avis depuis. Comme les anciennes couvertures de nos grands-mères, ces courtepointes critiques se révèlent indispensables aussitôt qu'on a goûté à leurs mérites: souvent belles, toujours utiles, preuves éloquentes que pour couvrir le corps... textuel, rien ne remplace le livre: la totalité est plus que la somme de ses parties. 
Le livre d'André Brochu rassemble des études publiées entre 1974 et 1990: Elles portent sur un certain nombre de poètes (Rina Lasnier, Claude Gauvreau, Paul Chamberland, Fernand Ouellette, Michel van Schendel, Claude Beausoleil), de romanciers (Julien Bigras, MarieClaire Blais, Anne Hébert, André Major, Jacques Brault, Yves Thériault, Gabrielle Roy) et même sur un cinéaste (Arthur Lamothe) et un essayiste québécois (Ernest Gagnon). Les essais sont quelquefois fort courts: simples reprises, par exemple, de comptes rendus publiés dans Livres et Auteurs québécois. Dans ces cas, l'étude laisse le lecteur sur sa faim, déçu que l'auteur n'ait pas jugé bon de reprendre et d'étoffer (de rembourrer, pour être fidèle à l'image de la courtepointe?) ses remarques ou ses intuitions, toujours stimulantes. Un compte rendu qui, en quelques brèves pages, comme c'est le cas pour Les Épouvantails d'André Major, réussit à fixer le non-sens d'une quête, la nature particulière de la temporalité et des visions narratives dans le roman et, finalement, la subversion du récit par la chronique, aurait certes mérité de devenir étude plus étoffée. Autrefois (dans son premier livre d'essais, L'Instance critique, publié en 1974), André Brochu distinguait les "études" de ces " aperçus". Ce n'est plus le cas.

D'autres analyses ont plus d'ampleur, heureusement. Ainsi celles sur Rina Lasnier, Claude Gauvreau, Julien Bigras, Yves Thériault, Jacques Brault ou Gabrielle Roy. Les approches de l'œuvre sont variées: thématiques et rhétoriques dans le cas de la poésie, plutôt formelles (disons "structurales") dans le cas des récits. Ainsi, les virtualités de l'analyse thétorique sont pleinement mises à l'épreuve dans l'étude des premiers versets de * La Malemer* de Rina Lasnier, mais davantage sans doute dans l'étude casse-cou du lettrisme chez Claude Gauvreau, étude où sont aussi bien convoquées les comptines, le latin, les glossolalies que les pulsions de là sexualité! Par ailleurs, l'étude d'Agonie de Jacques Brault, de La Quête de l'ourse d'Yves Thériault, d'un schème organisateur chez Gabrielle Roy, d'un roman "primitif" de Julien Bigras, sont autant de mises à l'essai des divers éléments de l'analyse des récits (diégèse, discours narratif, schème narratif, temporalité, etc.). Et chaque fois se déploie une "lecture* exemplaire, qui colle au texte, qui en épouse les méandres, qui en dévoile la structure textuelle, qui en cherche "la logique intime et essentielle. (p. 8): «lecture travaillante, qui ne se distingue pas d'une écriture, ni d'une réflexion* (p. 12).

"Le lecteur bienveillant (il en est)", dira André Brochu dans sa brève réflexion sur le thème de la ville dans l'œuvre de Claude Beausoleil, pour désigner le lecteur qui ne s'arrête pas aux ridicules possibles d'un texte. J'aime bien l'expression. Dans ces études, André 
Brochu me semble l'exemple même du lecteur bienveillant: respectueux des œuvres, soucieux de les mettre en valeur, répugnant au blâme (que de délicates circonlocutions dans l'étude des romans de Bigras!), cherchant et trouvant l'éclairage le plus propice pour lireinterpréter l'œuvre qui s'offre à lui, pratiquant ce qu'il appellera à un autre moment une "écoute proprement critique, celle qui promeut la compréhension du texte par une démarche d'empathie ${ }^{3 .}$ (p. 189). Promouvoir la compréhension. Cette bienveillance, cette empathie, est, en somme, une forme supérieure d'intelligence, qui s'applique à comprendre, c'est-à-dire à embrasser tout autant qu'à appréhender. Ainsi, André Brochu me semble avoir tout à fait raison de parler, dans son Avant-propos, de son culte des ouvres, chaque texte étant un absolu, nimbé - le mot n'est pas trop fort - de sacré.

Peut-être l'a-t-on perçu, à la lecture des quelques citations qui émaillent ce qui précède: ces études, si elles abordent un certain nombre d'œuvres singulières, portent aussi et beaucoup sur l'exercice critique lui-même. Livre de méthode, en quelque sorte. Recueil qui enseigne le bon usage des œuvres littéraires, d'une part, mais qui, d'autre part, se livre aussi à de salutaires réflexions sur la critique: ses butś, ses méthodes, ses moyens, son utilité et ses devoirs (et son incompatibilité avec la * recherche " dite "scientifique ${ }^{4}$ ).

Ces réflexions constituent l'essentiel du propos des chapitres initial et final: "Le travail de la lecture " et "La critique face à elle-même ou heurs et malheurs de Sophie Todorov m. Le lecteur de Brochu ý retrouvera les positions fondamentales qui sont celles de ce critique depuis ses premiers essais. Les phrases suivantes sont de quelle année, de quel livre?

La véritable recherche en critique, c'est celle qui, par l'écriture, s'associe au mouvement créateur de l'œuvre littéraire et le met en rapport avec le savoir.

Comprendre l'œuvre, à ma façon, et à la lumière du savoir contemporain.

Le critique [est...] l'intermédiaire total, qui a repensé l'œuvre et qui assume le risque de l'expliquer, à l'aide de tout un savoir qui est celui du chercheur, sans doute, mais à l'aide, aussi d'intuitions courageuses, de raccourcis que seule l'écriture, celle de l'écrivain, rend possibles.

Car voilà bien pourquoi, en dernière instance, (comme disent les althussériens), la critique est affaire d'écriture, quelle que soit la quantité de savoir dont elle s'encombre. L'écriture peut seule suggérer tout ce que, de sens, recèle l'œuvre par la vertu de dispositifs dont on peut montrer la complexité. 
La première est - je prends la date des livres - de 1992 (Le Singulier pluriel, p. 228); la seconde de 1974'; la troisième de 19886; la dernière de $1980^{7}$. Répétitions et reprises? Signes, plutôt, d'une réflexion ininterrompue sur les conditions d'exercice d'une vocation exigeante. "Je m'interroge beaucoup sur ce qu'est la critique *, dira André Brochu dans sa lettre-analyse à Julien Bigras (p. 144). Il s'interroge, en effet, depuis ses premiers pas en critique. Toujours prompt à se mettre en question et même à douter de son entreprise, revenant toujours, par ailleurs, à un certain nombre de valeurs essentielles, présentes à l'origine, toujours vivaces: respect de l'œuvre, empathie intelligente, analyse méthodique et rigoureuse mais sans prétention de scientificité, écriture heuristique qui invente son objet en s'inventant. Admirable constance devant cet universel singulier qu'est l'œuvre.

\section{**}

Il n'est pas certain que Joseph Bonenfant partagerait toutes les positions d'André Brochu. Ils se rejoignent, toutefois, dans une même ferveur face à la littérature. Passions du poétique, dit sans ambages Joseph Bonenfant. Entendons passion comme * enthousiasme, émotion, subjectivité reconnue devant l'objet. Le pluriel du titre nous indique bien que, tout en étant devant la passion du philosophe (tendance d'une certaine durée, assez puissante pour dominer la vie de l'esprit, auraient sans doute dit Jolivet ou Lalande), nous sommes aussi en présence d'autre chose: les états affectifs ou intellectuels, bien réels, qui traduisent les intérêts successifs dans lesquels se concrétise la passion. Toute passion, en effet, est spécifique, tendue vers un objet extérieur qui puisse la combler. Toujours déçue, évidemment, car aucun de ces intérêts ne peut la satisfaire pleinement. D'où le pluriel, le terme désignant, par synecdoque, les objets successifs de cette tension dynamique.

Le volume de Joseph Bonenfant nous présente donc, en trois sections distinctes, les manifestations partielles mais complémentaires de sa forte passion pour la poésie: les méthodes, les œuvres, l'enseignement. "Le premier bloc de textes s'articule autour des analyses thématiques, sémiotiques et pragmatiques qui marquent mon cheminement en littérature $[. .$.$] ; la deuxième partie comprend un survol d'œuvres poé-$ tiques importantes dans notre histoire littéraire [...]; je n'ai pas répugné, dans la troisième partie, à fouler le sol très quotidien de l'enseignement à l'université - (p. 12-13). Triple objet passionnel, propre à l'universitaire, sans doute, qui, à ses emballements de lecteur, joint consubstantiellement ceux de la réflexion théorique et du partage avec d'autres. 
On ne s'étonnera pas d'une approche passionnée des méthodes, des théories, des problématiques. Le souvenir des querelles d'écoles pendant les années soixante et soixante-dix devrait nous rappeler qu'il s'agit du lieu par. excellence du débat littéraire passionnel. En effet, si nous reconnaissons assez volontiers à chacun le droit à ses affinités électives pour ce qui est des œuvres, nous n'acceptons guère, par ailleurs, que notre propre façon d'aborder les œuvres et de les lire soit mise en cause, ou ne soit pas reconnue, sinon comme la seule valable; du moins comme la meilleure. Or, les quatre études de la première partie portent, à des degrés divers, les traces de cette passion exclusive.

Ainsi, un premier chapitre, inédit, effectue un excellent survol historique de la méthode thématique et se livre à une réflexion fort opportune sur ses acquis et ses limites. (André Brochu, d'ailleurs, est une référence constante dans l'étude, au même titre que les plus grands critiques français.) La nécessité de dépasser le thématisme y est affirmée avec force, en même temps qu'est reconnu le fait (et le paradoxe) que seuls les grands thématiciens soient capables d'effectuer ce dépassement. Le leitmotiv du chapitre, par ailleurs, est certainement l'absolue nécessité de la réflexion théorique, sous peine de se condamner à une naïveté critique désolante.

Les chapitres suivants approfondiront le bagage théorique indispensable à l'étude de la poésie, bagage qui, une fois acquis, deviendra connaissances pratiques, se transformera en actes et en capacités. La théorie, en somme, deviendra une *empirie. (p. 50). Une analogie amusante est même proposée pour contrer l'accusation de verser ou de se vautrer dans la théorie: c'est l'accusation que ferait un écolier de deuxième année, qui sait lire, à un écolier de sixième année, qui, en plus de savoir lire, est capable d'analyse grammaticale. "Je peux regarder un tableau ou un film sans la moindre initiation technique; ma fascination n'en sera que plus grande. Mais quand même, mais quand même!* (p. 35) s'exclame l'auteur. Que les critiques et les professeurs, én somme, soient au moins aussi savants que les poètes de leur temps!

Or, cette science, dans l'esprit de Joseph Bonenfant, est essentiellement une "conscience linguistique" avertie et ambitieuse (chapitre II). C'est-à-dire, la capacité de faire un inventaire complet du plan de l'expression d'un texte, puisque tout signifie dans le poème et que rien, "mais absolument rien. (p. 37) n'est négligeable. Cette conscience linguistique conduit logiquement à une pragmatique (chapitres III et IV), une interrogation de l'énonciation dans le discours poétique, c'est-à-dire la volonté de "constamment resituer l'énoncé 
poétique dans son contexte d'énonciation et dans son effet de fiction. (p. 42). Est ainsi proposé .l'examen descriptif des moyens discursifs, des moyens stratégiques utilisés en vue du succès de la communication . (p. 50), examen inséparable de "l'arrière-fond sociohistorique" (p. 60) sur lequel le discours poétique prend appui.

Il ne faudrait pas croire, par ailleurs, que ces propositions théoriques, articulées avec force et conviction, tombent dans un exclusivisme étroit. (Comment peut-on partáger les passions d'autrui? se demandait d'ailleurs Joseph Bonenfant dans l'Introduction.) Certes, à certains moments dans les études qui suivent, l'auteur verse dans ce qu'on pourrait qualifier de zèle excessif pour la théorie. Deux études sur Fernand Ouellette, par exemple, mettent curieusement en cause l'ensemble de la critique littéraire existante. "Discours interminablement long ", "discours paraphrastique. (p. 105), "discours anciens... très dichotomisés, parcellaires, approximatifs. (p. 117), dira l'auteur, pour leur opposer, en général, la théorie littéraire, qui * crée plutôt des procédures descriptives aptes à vérifier l'efficacité littéraire•(p. 105) et, en particulier, le structuralisme: "́la voie structurale ouvre sur la plus large et la plus profonde opération de lecture qu'on puisse imaginer * (p. 117). Mais ces études, on l'aura deviné, sont de 1972 et 1973, période propice aux formulations de ce genre et aux enthousiasmes qui déchanteront.

La plupart des études, au contraire de celles-ci, comme les réflexions nées d'une pratique pédagogique diversifiée, nuancent plutôt le zèle théorique et pratiquent un éclectisme large mais rigoureux. Les analyses qui mettent en évidence, successivement, la modernité de Nelligan, le caractère narratif et fondateur de la poésie grandboisienne, l'aspect réflexif (mallarméen) des poèmes de Saint-Denys Garneau et de Miron, la dimension iconique de l'œuvre de Rina Lasnier, la thématique de la lumière et de la violence chez Jean-Guy Pilon; la visée totalisante de l'œuvre de Gatien Lapointe, la féminité juive et souffrante de Monique Bosco, la quotidienneté chez Michel Beaulieu, le dénuement face au monde, à soi, à l'écriture dans l'œuvre de' François Charron, sont autant d'ouvertures larges et profondes sur de riches univers poétiques. Les dernières études du livre, d'ailleurs (qui sont aussi les dernières chronologiquement), en viennent même à contester ce qui vingt ans auparavant était article de foi. Ainsi, les lectures sérielles effectuées sur l'œuvre de Charron sont présentées comme n'ayant * rien à voir avec de stériles gerbes thématiques ou découpages structuraux (p. 184). Est-ce une façon de contester la *lecture structurale d'un poème de Fernand Ouellette. (p. 115-132), commise vingt ans plus tôt? 
En partie, sans doute. Chaque attachement passionné chasse le précédent, c'est bien connu. Mais, sans doute, avec l'expérience, un phénomène de lassitude se fait-il jour. Joseph Bonenfant peut même en venir à parler d'un *marché institutionnel * des théories, étalées en quelque sorte devant le consommateur universitaire.

Depuis quelques années, on invente des théories de la littérature à partir de l'avènement des diverses linguistiques, sémiotiques et autres pragmatiques, à partir de concepts sociologiques et psychanalytiques. Ne risquet-on pas de connaître une mode qui, un jour, réduira l'enseignement de la littérature à l'histoire de ses théories et de ses critiques, sinon aux théories et à la critique de son histoire? (p. 198)

Le ton, on le constate, est quelque peu ironique et désabusé (et tout à fait analogue à la distance critique de Brochu devant les théories et leurs prophètes). Est-ce la sagesse-ou la quiêtude qui succède à la passion? Peut-être. Mais peut-être est-ce aussi souci d'intégration. Car en même temps qu'une passion chasse la précédente, elle l'intègre et l'assume.

Et, en effet, on s'aperçoit, à la lecture de ces analyses 'et de ces réflexions, que chacune est l'indice d'une évolution, d'un parcours, marqué au coin de l'accueil et de l'ouverture. La visée quelque peu totalisatrice des chapitres théoriques (elle-même bien relative, dans le contexte de l'époque). est singulièrement assouplie quand vient le moment d'étudier les œuvres. Parlant de son travail pédagogique en atelier, Joseph Bonenfant formule l'attitude qui me semble aussi celle du critique: «Étant férocement contre la démolition hystérique, je suis parfois le premier à trouver quelque chose de bon quelque part, sous un certain angle et sous certaines coutures.- (p. 203) L'attitude du professeur devant ses étudiants est en quelque sorte celui du théoricien et du critique. Ouverture et générosité, qu'on aurait tort de confondre avec une bienveillance mollasse. La bienveillance, on l'a vu avec André Brochu, est partie intégrante de l'intelligence. "Il faut cependant qu'à une ouverture très grande corresponde une exigence très stricte." (p. 212) Le maximum de l'accueil et de l'exigence, voilà en effet ce qu'on trouvera dans ce livre attentif à toutes les dimensions du poétique.

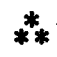

Dans son Avant-propos, André Brochu s'interrogeait sur *la masse énorme de ce qui ne sera jamais lu. et y voyait un indice troublant de l'agonie de la littérature. Celle-ci, en effet, avait cessé d'être la référence culturelle par excellence et pourtant la machine produisait toujours de 
plus en plus de livres. * Loin d'être vide, la maison Littérature meurt de ce qui l'encombre. . Si la critique est le culte des œuvres et la coïncidence (au sens géométrique) passionnée avec elles, comment s'attendre du lecteur, en effet, qu'il se partage à l'infini. Tous ces livres ne deviendront qu'objets, qui joncheront, par centaines, le sol.

Réfléchissant sur sa pratique pédagogique en ateliers d'écriture, Joseph Bonenfant en vient à la même constatation mi-désabusée, mialarmée. *Au Québec, comme dans l'atelier, ce n'est pas l'écriture qui manque, mais la lecture.. La lecture. Faire du livre un objet vivant, un objet-sujet, indissociable de soi et de la mémoire collective.

Mais pour cela il faut se colleter au texte. Comme font ces critiques. Entreprise hasardeuse, difficile, et, cela va de soi, impossible à comptabiliser. Comme la passion. Comme la vie. Comme le véritable savoir.

1. André Brochu, Le Singulier pluriel. Essais, Montréal, L'Hexagone, coll. -Essais littéraires :, 1992, $234 \mathrm{p}$.

2. Joseph Bonenfant, Passions du poêtique, Montréal, L'Hexagone, coll. «Essais littéraires :, 1992, $234 \mathrm{p}$.

3. Bienveillance qui ne semble pas s'étendre, toutefois, jusqu'aux théoriciens jargonneurs! À preuve cette parodie du kristevisme: . En termes élégamment kristeviens, on avancera plutôt que le phéno-texte se soutient de l'irruption en lui de la chôra sémiotique, laquelle y fait plus qu'insister, s'arroge tout l'espace du symbolique, le réduit à la manifestation la plus ténue. (Peut-être est-ce encore un peu simplet).

4. - La Recherche, c'est cette institution universitaire qui, à mon avis, cause le plus grand tort à la critique en l'obligeant à renier sa vocation proprement littéraire au profit d'une orthodoxie pseudo-scientifique. - (p. 227)

5. André Brochu, s. La critique en question:, L'Instance critique 1961-1973, Montréal, Leméac, 1974, p. 85.

6. Id., . Critique et recherche , La Visée critique, Montréal, Boréal, 1988, p. 141.

7. André Brochu et Gilles Marcotte, La Littêrature et le reste (livre de lettres), Montréal, Quinze, 1980, p. 167. 